\title{
ERRATA
}

\section{Erratum: "Nonlinear optical response of GaN layers on sapphire: The impact of fundamental beam interference"' [Appl. Phys. Lett. 76, 810 (2000)]}

I. V. Kravetsky and I. M. Tiginyanu

Institute of Applied Physics, Technical University of Moldova, 2004 Chisinau, Moldova

Ralf Hildebrandt and Gerd Marowsky

Laser-Laboratorium Göttingen e. V., Hans-Adolf-Krebs-Weg 1, D-37077 Göttingen, Germany

D. Pavlidis and A. Eisenbach

Solid State Electronics Laboratory, The University of Michigan, Ann Arbor, Michigan 48109-2122

H. L. Hartnagel

Institut für Hochfrequenztechnik, Technische Universität Darmstadt, D-64283 Darmstadt, Germany

[S0003-6951(00)09910-1]

Due to a production error, Eq. (5) appeared incorrectly. The correct equation is:

$d_{\mathrm{eff}}^{p-p}=d_{15} \cos \theta_{2 \omega} \sin 2 \theta_{\omega}+d_{31} \cos ^{2} \theta_{\omega} \sin \theta_{2 \omega}+d_{33} \sin ^{2} \theta_{\omega} \sin \theta_{2 \omega}$. 